\title{
HPV vaccination-reaping the rewards of the appliance of science
}

This Editorial (BMJ 2013;346:f2184, doi:10.1136/bmj.f2184) contains an error in the last sentence of the third paragraph. This sentence should have read: "From 2007 to 2011, there was no significant decrease in the prevalence of genital warts in heterosexual men over 30 [not 21, as was published] years or in men who have sex with men."

Cite this as: BMJ 2013;347:f5129

๑ BMJ Publishing Group Ltd 2013 